\title{
Coordinated Damping Control Through Multiple HVDC Systems: A Decentralized Approach
}

\author{
Yousef Pipelzadeh, Student Member, IEEE, Balarko Chaudhuri, Member, IEEE, Tim C. Green, Senior \\ Member, IEEE
}

\begin{abstract}
Role of multiple HVDC links - both CSC and VSC - in improving the AC system dynamic performance is presented here. An Australian equivalent system with three critical interarea modes is considered to illustrate the case study on DIgSILENT PowerFactory. Subspace-based multi-input-multi-output (MIMO) system identification is used to estimate and validate linearized state-space models through pseudo random binary sequence (PRBS) probing in DIgSILENT PowerFactory. Choice of appropriate input-output combinations is done through modal residue analysis taking note of not only the magnitudes but also the phase angles. The decentralized control design is posed as an optimization problem and solved using an evolutionary technique. The results are validated through linear analysis and non linear simulation in DIgSILENT for two extreme (light and heavy loading) operating conditions.
\end{abstract}

Index Terms-HVDC, Current source converter (CSC), Voltage source converter (VSC), Stability, Modal analysis, Identification, Decentralized control.

\section{INTRODUCTION}

O $\mathrm{NE}$ of the major concerns in power transmission systems is secure and stable system operation, especially, in the presence of low-frequency electro-mechanical oscillations $(0.1-1.0 \mathrm{~Hz})$. These oscillations are often poorly damped, especially with increasing power transfer levels [1].

The occurrence of these low-frequency swing oscillations are due to dynamic interactions between groups of synchronous machines interconnected by long, relatively weak inter-ties in large systems. Their damping is influenced by automatic voltage regulators (AVRs) with high gains in the generators and long transmission corridors establishing weak connections between different regions. Modulation of High Voltage Direct Current (HVDC) can be extremely effective for damping power oscillations and thereby increasing the transfer capacity limits of an AC transmission system [2].

Existing projects such as the Pacific DC Inter-tie (which forms part of the Western US power system) focus on HVDC control to damp oscillations based on CSC technology. This is of course a well established and reliable technology. However, it imposes controllability limitations in the sense that only the active power flow can be modulated. A VSC HVDC link allows independent modulation of both the active power and reactive power (or voltage) at either ends and hence provides more flexibility than CSC. With ever increasing power transfer

\footnotetext{
Support from the EPSRC UK under grant EP/F037686 (Power Networks Research Academy) is gratefully acknowledged.

The authors are with the Control and Power Research group, Imperial College London, London, UK e-mail: \{y.pipelzadeh08,b.chaudhuri, t.green\}@imperial.ac.uk
}

levels, many systems will include multiple HVDC links. There is tremendous potential for coordinated damping control through CSC \& VSC HVDC to contribute towards improving the AC system dynamic conditions.

In recent years there has been vast amount of interest into the application of decentralized control techniques to design damping control devices in power systems. A number of approaches to decentralized control have been proposed. However, the focus of most of them are on decentralized design of low-order PSS in an SISO or Multiple-InputMultiple-Output (MIMO) framework; or otherwise, on the robust control design techniques which evidently result in high order controllers with practical limitations. Ramos et al. [3] used dynamic output feedback for decentralized design using Linear Matrix Inequality (LMIs) considering multiple operating conditions. The controller order, however, is at least, as high as the open-loop plant. Messina et al. [4] used a decentralized approach to co-ordinate multiple Flexible AC transmission system (FACTS) devices using classical control approach, however, it may lack robustness with varying operating conditions.

This paper demonstrates the design of a low-order, MIMO decentralized controller through multiple HVDC devices to provide adequate damping of inter-area modes across multiple operating conditions.

The contributions of this paper are:

1) Steady-state and dynamic modelling of both CSC and VSC based HVDC systems in DIgSILENT.

2) The use of subspace based MIMO system identification to estimate and validate state-space models by injecting PRBS probing signals into an IEEE benchmark test system.

3) Demonstrate decentralized design in a MIMO framework to damp multiple swing modes using multiple CSC/VSC HVDC device installed in an Australian equivalent system.

4) Modal analysis substantiated by non-linear simulations in DIgSILENT PowerFactory to validate the findings.

\section{HVDC CONTROLLER Modeling}

\section{A. HVDC Technologies}

The two main HVDC transmission technologies currently in operation are the current source converter (CSC) and the more modern voltage source converter (VSC). With the development of reliable and more flexible self-commutating conversion, 
CSC technology is facing increasing competition from VSCHVDC technology with many suppliers such as ABB, Siemens and ALSTOM providing low to medium power rating solutions. VSC has a number of major advantages over CSC. These include the ability to modulate (control) the active \& reactive power at both ends of the link independently and hence offers more flexibility than CSC where only the active power can be modulated. Despite the limited controllability of CSC, they are still greater used in high power transmission fields and is superior to VSC transmission in terms of capital cost, power losses and reliability for large scale HVDC transmission.

\section{B. Control of the CSC-HVDC link}

Contrary to the VSC-HVDC, the CSC-HVDC has only one free control variable, the firing angle $\alpha$. This signal influences the active power transferred by the HVDC link. Since it is not possible to control the required reactive power; it can only be limited with a small excitation angle.

The main objective of the CSC-HVDC is to control the active power transmission. Earlier HVDC schemes adopted current current loop as the basis for operation whereby each station was equipped with a divider circuit with $P_{\text {ord }} \& U_{d c}$ signals used to derive the current order, $I_{\text {ord }}$. A control error is formed by comparing the calculated $I_{\text {ord }}$ with a current margin (set to 0.1 ) and the measured $I_{D C}$. There also exists a master controller which sends the $P_{\text {ord }}$ command to the pole controls of both HVDC link ends. As emphasized in Section II.A, the interest of this paper is on the supplementary modulation (control) which acts through the master control level.

In normal operation the rectifier controls the set-point active power or direct current and the inverter controls the DC link voltage. The control scheme for the rectifier converter is shown in Fig. 1. It includes a current control with voltage dependent current order limit (VDCOL) used to reduce the current setting when the voltage decreases. Fig. 2 shows the control functions used in DC voltage and constant extinction angle at the inverter.

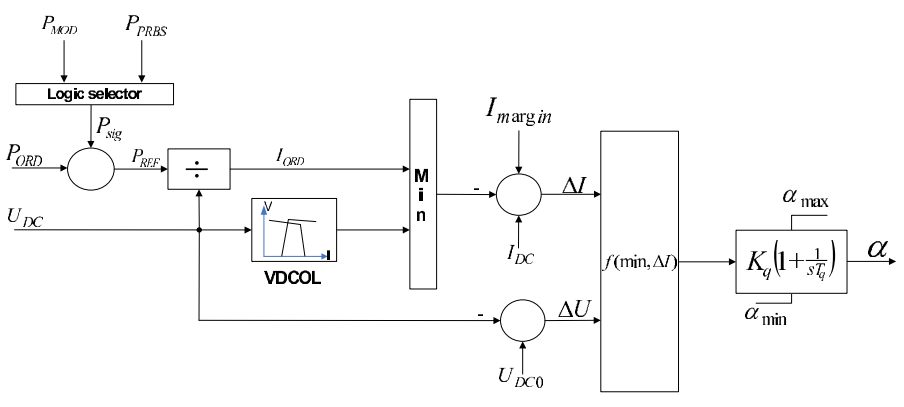

Fig. 1. Control scheme for CSC-HVDC converter (firing angle control)

\section{Control of the VSC-HVDC link}

The VSC converter model represents a self-commutated, voltage sourced $\mathrm{AC} / \mathrm{DC}$ converter with sinusoidal modulation. The model of all steady-state functions and RMS-simulations are represented by the fundamental frequency approach. At fundamental frequency, the ideal, loss-less converter can be

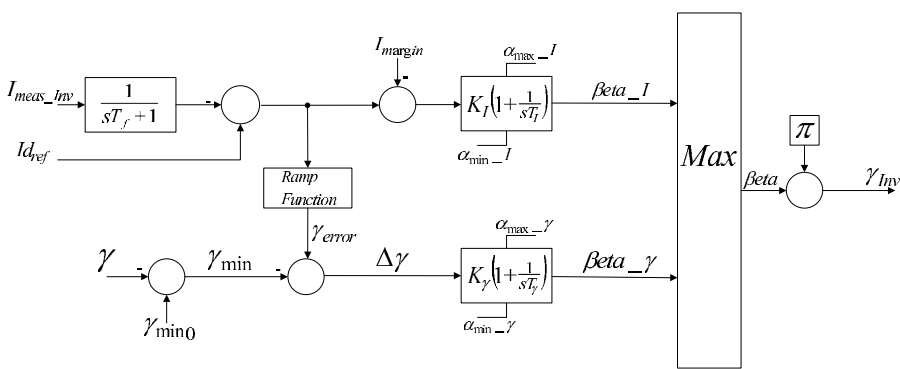

Fig. 2. Control scheme for CSC-HVDC converter (extinction angle control)

modelled by a DC-voltage controlled AC-voltage source conserving active power between AC- and DC-side. Depending on the application, different load-flow and stability control conditions can be specified. A very common application is to measure the voltage angle using a phase locked loop (PLL) and to connect the output of a $d q$-current controller to Pmd and $P_{m q}$. Current control approach in the $d-q$ reference frame is adopted for the primary pole control loops due to its advantages over voltage control strategy[5]. The control for the VSC HVDC scheme is presented in Figs 3 and 4.

The AC system connected to the VSC-HVDC grid is modeled in $d-q$ reference frame which needs to be transformed to $d^{\prime}-q^{\prime}$ frame and back while interfacing the VSC-HVDC grid variables with those of the AC system. The dynamics of the $\mathrm{AC}$ side of the converters is given by:

$$
\begin{gathered}
v_{d t}^{\prime}-R_{c} i_{d}^{\prime}-L_{c} \frac{d i_{d}^{\prime}}{d t}+L_{c} \omega_{e l} i_{q}^{\prime}=E_{a c} \\
v_{q t}^{\prime}-R_{c} i_{q}^{\prime}-L_{c} \frac{d i_{q}^{\prime}}{d t}-L_{c} \omega_{e l} i_{d}^{\prime}=0
\end{gathered}
$$

where, $v_{d t}^{\prime}=m_{d}^{\prime} \frac{U_{d P} P}{2}, v_{q t}^{\prime}=m_{q}^{\prime} \frac{U_{d P}}{2}$ and $R_{c}, L_{c}$ are the aggregated resistance and inductance of the converter transformer and phase reactors.

The DC bus dynamics at each converter end was modeled by the power balance in the capacitors. The converter DC bus model as written below:

$$
P^{\prime}-P-i^{\prime 2} R_{c}=\frac{1}{2} C_{s_{-} p} \frac{d\left(U_{d}^{2} P\right)}{d t}
$$

1) Converter $P-Q$ Control: The sending end converter station is considered to operate in $P-Q$ control mode as shown in Fig. 3. The reference values of the current components were derived as:

$$
i_{d}^{\prime *}=\frac{2 P^{*}}{3 E_{a c}}, i_{q}^{\prime *}=-\frac{2 Q^{*}}{3 E_{a c}}
$$

2) Converter $U_{d c}-Q$ Control: The receiving end converter station operates in $U_{d c}-Q$ control mode maintaining constant DC bus voltage and unity power factor at the connection point as shown in Fig. 4.

\section{Test System In DIgSILENT}

\section{A. Australian Equivalent Network}

A 14-machine, 59-bus equivalent of the Australian network (shown in Fig. 5) was considered for the case study. This system has been adopted as an IEEE benchmark for stability 


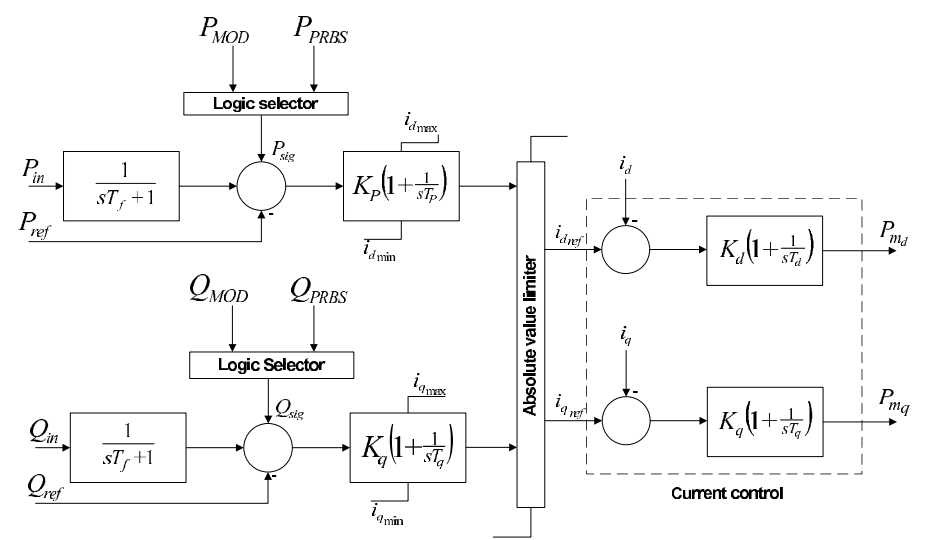

Fig. 3. Control scheme for VSC-HVDC converter (sending end)

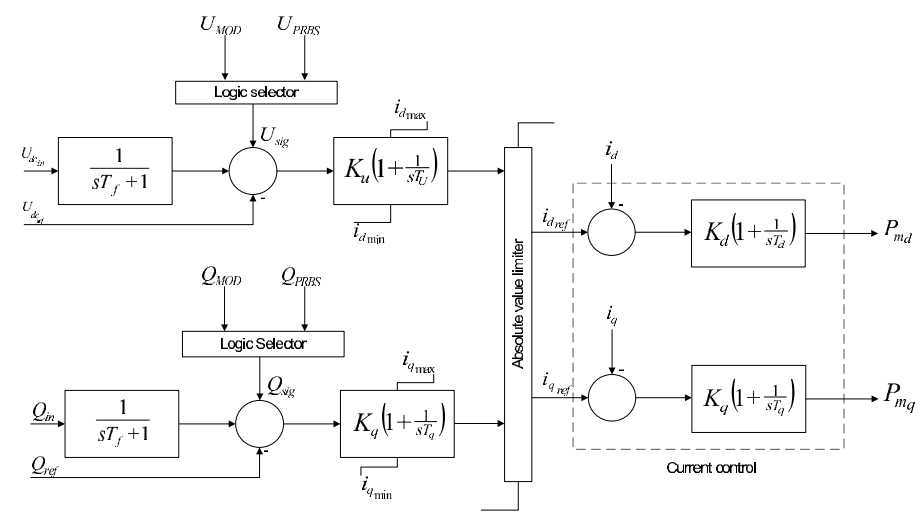

Fig. 4. Control scheme for VSC-HVDC converter (receiving end)

studies. The detailed description of the study system including network data and dynamics data for the generators, excitation systems, PSSs and SVCs can be obtained from [6]. The network consists of 5 areas in which areas 1 and 2 are closely coupled electrically. Therefore, in essence the power system is made up of 4 geographical regions and has 3 inter-area modes beside 10 local modes.

The machines are equipped with either IEEE type AC1A, AC4A or ST5B excitation system models. All generators have speed-input power system stabilizers of second and fourth order transfer functions. Five Static VAr Compensators (SVCs), set in voltage control mode, are installed (1 in each area), to improve the voltage profile of the system. The loads are all assumed to be of Constant Impedance (CI) type for all operating conditions.

\section{B. Problem Description}

Power flow and dynamic analysis for multiple loading scenarios have been benchmarked and validated against the standard results reported in [6]. The impact of both VSC and CSC based HVDC transmission technologies (after detailed modelling in DIgSILENT) with only their primary controls on the overall small-signal stability has been assessed in the above benchmark test system. It became evident that the boundary flows between regions of the network are highly stressed due to stability limit related to inter-area oscillation between generator groups. Fig.6. illustrates the 5-area test system with HVDC upgrades, including a VSC-HVDC link in parallel to

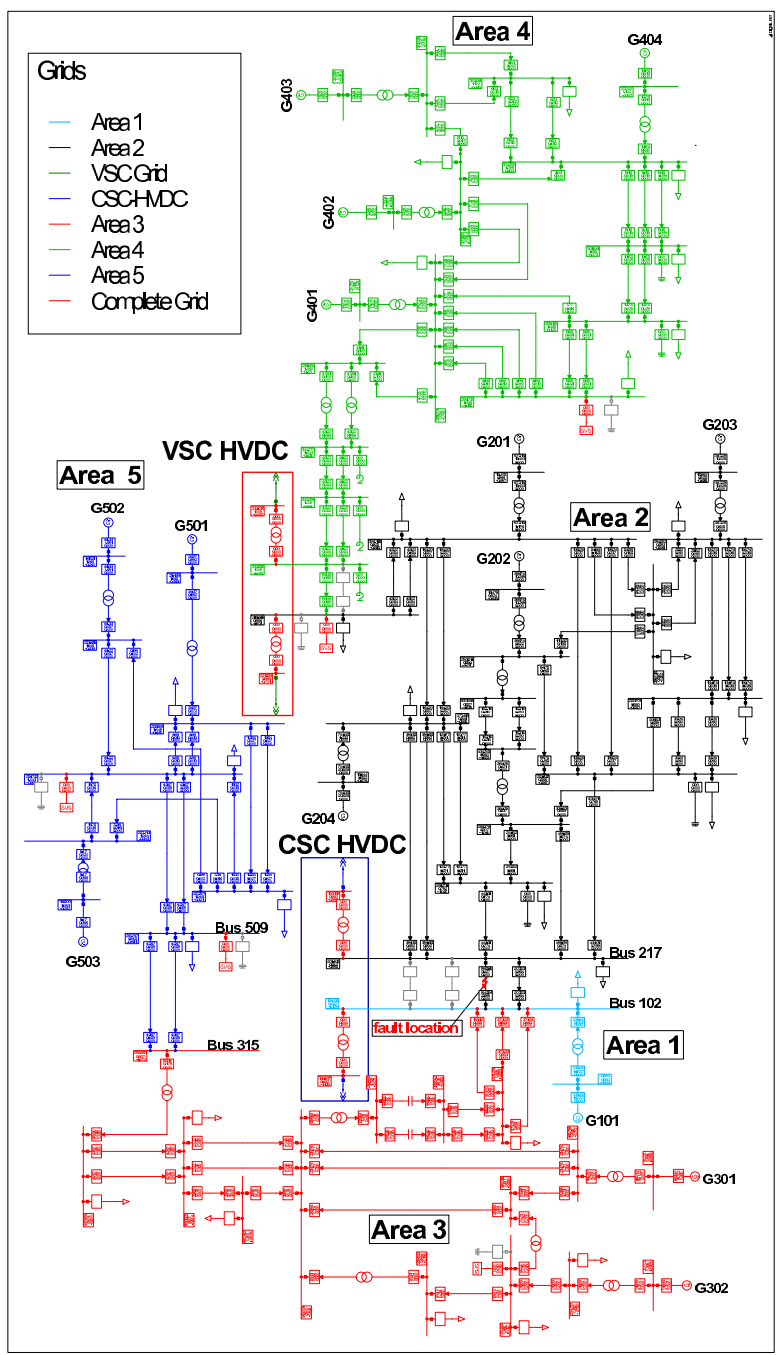

Fig. 5. 14-machine, 5-area Australian equivalent network modelled in PowerFactory DIgSILENT.

the AC corridors between area 2 and area 4 and a CSC- HVDC link between area 1 and area 2 .

The primary control loop parameters for the CSC- HVDC (as shown in Figs. 1 and 2) are based on the CIGRE benchmark model [7] with ratings of $\pm 500 \mathrm{kV}, 1000 \mathrm{MW}$. The VSC is a bipolar link with ratings of $\pm 150 \mathrm{kV}, 350 \mathrm{MW}$. The DC links are commanded to share $50 \%$ of the total inter-area flows through the respective corridors(originally accounted by the AC lines but now placed out of service). Out of several scenarios considered only the heavy loading and light loading scenarios are presented here (see Table I). The reactive power order was set to maintain close to unity power factor at the $\mathrm{AC}$ terminals.

\section{Modal Analysis}

To demonstrate power oscillation damping (POD) control through multiple HVDC links, 6 out of the 14 PSSs were placed out of service to simulate a more oscillatory behaviour. Modal analysis was performed for the two extreme loading conditions to identify the damping and frequency of the system eigenvalues. As seen from Figs 7 and 8, there are 3 poorly 


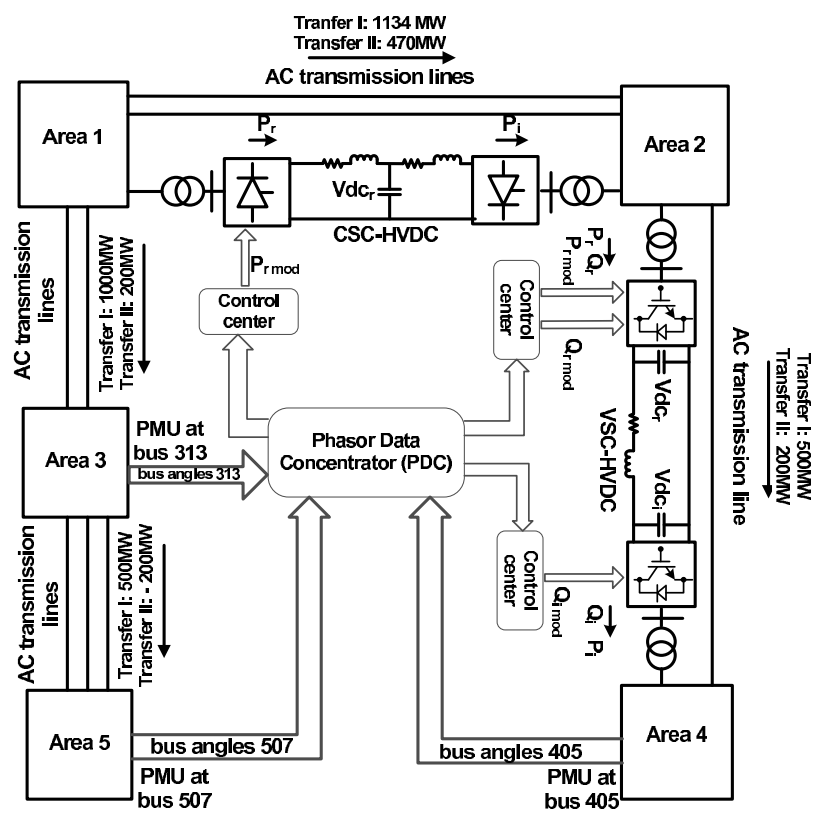

Fig. 6. 14-machine, 5-area test system with CSC-HVDC and VSC-HVDC upgrades. Secondary control loops with PMU signals are shown

TABLE I

TOTAL GENERATION AND INTER-TIE TRANSFERS FOR TWO LOADING CONDITIONS

\begin{tabular}{|c||c||c|}
\hline \multicolumn{1}{|c||}{} & Heavy Loading & Light Loading \\
\cline { 2 - 3 } & \multicolumn{2}{|c|}{ Active Power (MW) } \\
\hline \hline Generation & 23030.1 & 15045.9 \\
\hline Load Supply & 22300 & 14810 \\
\hline \hline Inter-area tie flow & \multicolumn{2}{|c|}{} \\
\hline \hline Area 2 to Area 1 & $\mathbf{1 1 3 4 . 3}$ & $\mathbf{4 6 9 . 7}$ \\
\hline Area 1 to Area 3 & 1001.5 & 199.7 \\
\hline Area 4 to Area 2 & 500.7 & -200.3 \\
\hline Area 3 to Area 5 & 499.9 & 199.9 \\
\hline
\end{tabular}

damped modes. This sets the motivation for developing a control scheme to improve the dynamic stability of the system under both extreme loading conditions.

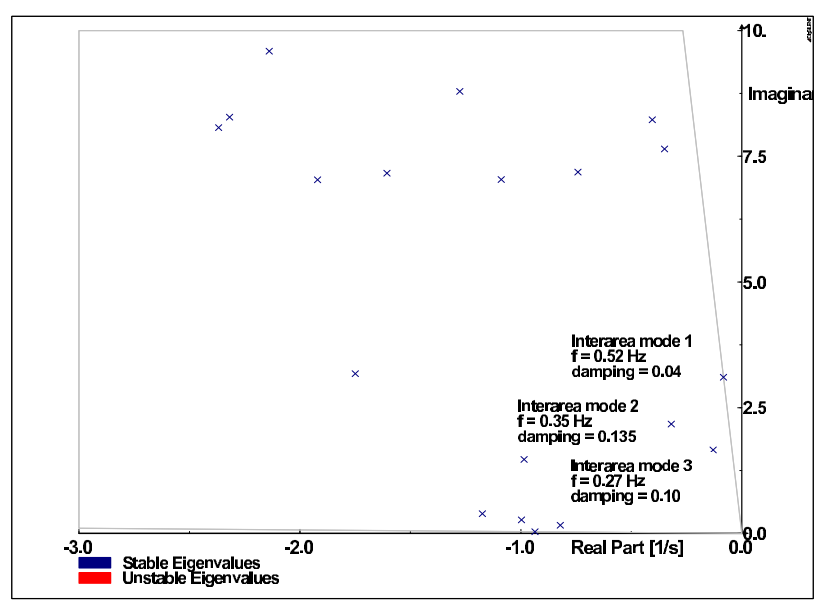

Fig. 7. Modal analysis for the heavy loading scenario (includes both interarea and local modes)

The objective is to illustrate the ability of the HVDC links to provide services that enable optimized power flow and

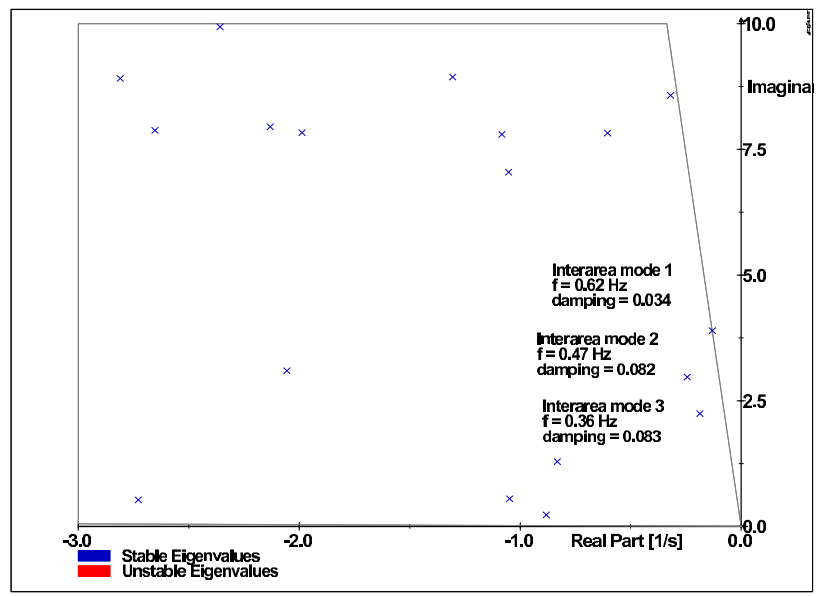

Fig. 8. Modal analysis for the light loading scenario (includes both inter-area and local modes)

stabilizing services (through control centers) for extending the stability limit of the AC network. The next section introduces the methods applied to improve the damping of the system to ensure stable system operation.

\section{MiMO Linear Model Estimation AND VALIDATION}

Input (B) and Output $(C)$ matrices of the linearized statespace model cannot be directly obtained from DIgSILENT. Therefore, a system identification technique was used to estimate the linear model from the simulated outputs in response to appropriate probing signals at the inputs. Here, the following 4 control inputs were chosen: $P_{r}$ for the CSC HVDC and $P_{r}, Q_{r}$ and $Q_{i}$, for the VSC HVDC. Phase angle measurements available from the PMUs at 16 locations were considered. Identification of such MIMO systems is quite challenging and gets further complicated with increase in number of output signals [8].

\section{A. Probing Signal}

Selection of an appropriate probing signal plays an important role in system identification. Different probing signals, such as repeated pulses, band-limited gaussian white noise, PRBS, Fourier sequence, have been reported in the literature [9]. Here PRBS was chosen due to its richer spectrum. PRBS amplitude was chosen to be high enough to sufficiently excite the critical modes without pushing the responses into predominantly nonlinear zones. Moreover, the probing sequence for different inputs were ensured to be uncorrelated [8]. Typical PRBS injection signals used for probing the test system are shown in Fig. 9.

\section{B. MIMO Subspace Identification}

The discrete state space model of the MIMO system with $m$ inputs and $l$ outputs can be expressed as:

$$
\begin{aligned}
& x(k+1)=A_{d} x(k)+B_{d} u(k)+w(k) \\
& y(k)=C_{d} x(k)+D_{d} u(k)+v(k)
\end{aligned}
$$




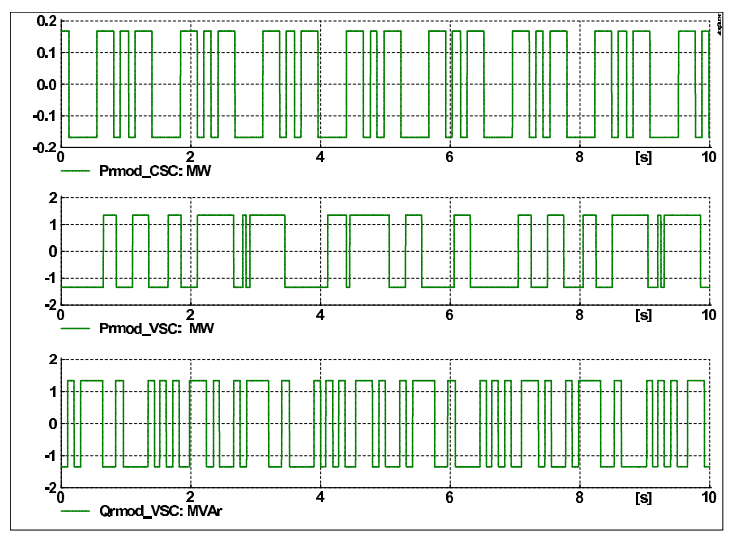

Fig. 9. Typical PRBS injection used for MIMO system identification

where, $A_{d} \in \Re^{n \times n}, B_{d} \in \Re^{n \times m}, C_{d} \in \Re^{l \times n}$ are the matrices to be estimated and $w(k) \in \Re^{n \times 1}$ and $v(k) \in \Re^{l \times 1}$ are nonmeasurable observation and process noise vector sequences. Here, the input vector $u(k) \in \Re^{4 \times 1}$ is the set of PRBS injection signals at the 4 control inputs of both HVDC links and the simulated output responses $y(k) \in \Re^{16 \times 1}$ are the phase angles of voltage measured from the 16 PMUs.

Using the input probing signal $\left\{u_{i}(0), u_{i}(1), \ldots u_{i}(N), i=\right.$ $1,2, \ldots 4\}$ and output responses $\left\{y_{i}(0), y_{i}(1), \ldots y_{i}(N), i=\right.$ $1,2, \ldots 16\}$ the matrices $A_{d}, B_{d}, C_{d}$ and $D_{d}$ were calculated such that the simulated (actual) data matched the responses from the estimated (identified) linear model. The estimated model in discrete domain was converted to continuous domain for linear analysis and control design. N4SID, a function in system Identification Toolbox of MATLAB [10] was used to estimate the above matrices. A model order of 30 was found to be appropriate for both heavy and light scenarios.

\section{Model Validation}

To validate the estimated linear model of the MIMO system, pulses of $0.5 \mathrm{~s}$ duration were applied at the 4 control inputs separately and in all possible combinations. The blue (black in grey-scale) traces in Fig. 10 show the simulated responses from DIgSILENT against the red (grey in grey-scale) ones obtained from the identified linear model. Very close match between the red and blue traces in all cases (only a few representative cases are shown here) confirms the accuracy of the estimated linear models.

\section{Feedback Signal Selection}

\section{A. Residue Analysis}

Residues provide a combined measure of controllability and observability of the modes of interest of a linearized system model which can be expressed in state-space form as [1]:

$$
\left[\begin{array}{l}
\Delta \dot{x} \\
\hline \Delta y
\end{array}\right]=\left[\begin{array}{l|l}
A & B \\
\hline C & D
\end{array}\right]\left[\begin{array}{l}
\Delta x \\
\hline \Delta u
\end{array}\right] ; G(s) \triangleq\left[\begin{array}{c|c}
A & B \\
\hline C & D
\end{array}\right]
$$

Applying an appropriate transformation, (6) can be transformed into a modal (normal or decoupled) form as:

$$
\left[\begin{array}{c}
\dot{z} \\
\hline \Delta y
\end{array}\right]=\left[\begin{array}{c|c}
\Lambda & \Phi^{-1} B \\
\hline C \Phi & D
\end{array}\right]\left[\begin{array}{c}
z \\
\hline \Delta u
\end{array}\right]
$$

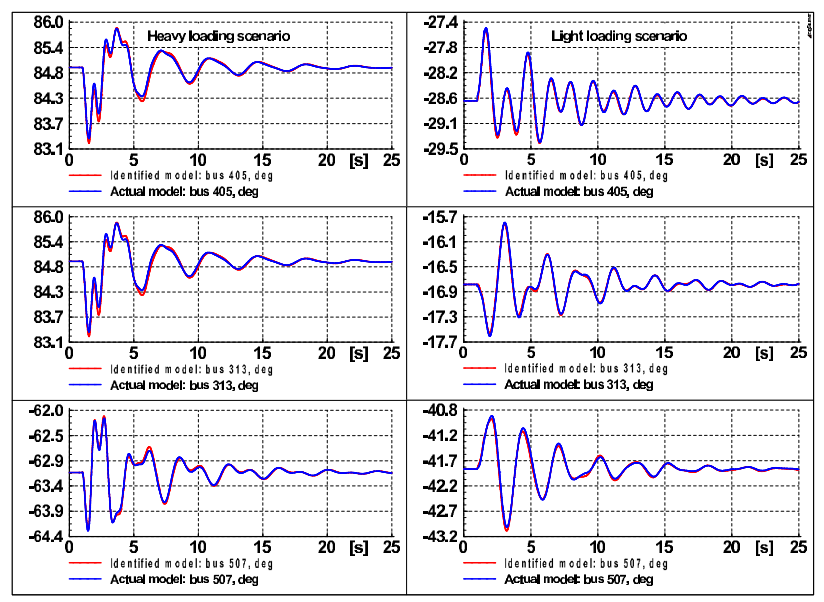

Fig. 10. Validation with a pulse excitation on the linear model and actual non-linear system for both heavy and light loading conditions. Legends below the plots show the variables plotted

where $\Lambda=\Phi^{-1} A \Phi$ is a diagonal matrix and $\Phi=$ $\left[\begin{array}{llll}\phi_{1} & \phi_{2} & \ldots & \phi_{n}\end{array}\right]$ is the right eigen vector (or modal) matrix comprising the right eigen vectors $\left(\phi_{1}, \phi_{2}, ..\right)$ corresponding to each mode.

Modal observability $O b_{i j}$ of the $i^{\text {th }}$ mode in the $j^{\text {th }}$ output is obtained by multiplying the $j^{\text {th }}$ row vector of $C$ with the $i^{\text {th }}$ column of $\Phi$. Similarly, modal controllability $C o_{i k}$ of the $i^{\text {th }}$ mode in the $k^{\text {th }}$ control input is the product of the $j^{\text {th }}$ row vector of $\Phi^{-1}$ and the $k^{\text {th }}$ column of $B$.

$$
\begin{aligned}
C o_{i k} & =\left[\Phi^{-1}\right]_{i} \times[B]_{k} \\
O b_{i j} & =[C]_{j} \times[\Phi]_{i}
\end{aligned}
$$

The product of modal controllability and observability gives the residue $\operatorname{Res}_{i-k j}=C o_{i k} \times O b_{i j}$ which indicates the extent to which mode $i$ can be observed and controlled through input $k$ and output $j$. The elements of an eigenvector are complex numbers, in general, and as such modal controllability $\mathrm{Co}_{i j}$, modal observability $O b_{i k}$ and residue $R e s_{i-k j}$ are all complex numbers with a magnitude and a phase angle component.

Once the residues corresponding to all possible input-output combinations are calculated and sorted in descending order of magnitude, the appropriate ones are chosen from the top (with highest residues) to ensure minimum control effort. For SISO systems the phase angle of the residue is not important. However, for SIMO or MIMO systems, the phase angle could be critical when choosing appropriate inputs and outputs [11].

In this work a decentralized control framework is used with control (modulation) inputs: $P_{\text {rmod }}$ for CSC HVDC and $P_{\text {rmod }}, Q_{\text {rmod }}$ and $Q_{i m o d}$ for VSC HVDC, and 16 outputs (the phase angles measured by the PMUs installed at 16 chosen buses). Residues were calculated for all possible input-output combinations.

The objective here is to appropriately select input-output pairs such that an $m$-inputs and $m$-outputs decentralized controller is synthesis to provide adequate damping for multiple operating conditions. Three measured outputs - phase angles at buses $313,405,507$ corresponding to $P_{\text {rmod }}$ (CSC), $P_{\text {rmod }}$ (VSC), $Q_{\text {rmod }}$ (VSC) were found to be the most effective signals for the three critical inter-area modes for both heavy 
and light loading conditions.

\section{Power Oscillation Damping (POD) CONTROL}

\section{A. Design Specification}

The objective is to design a decentralized control in the MIMO framework to damp multiple swing modes through multiple HVDC devices. The controller is required to ensure that modal oscillations corresponding to each of the $n$ critical inter-area modes settle within $10.0-12.0 \mathrm{~s}$ under all the operating conditions.

\section{B. Decentralized Controller Synthesis}

The objective is to design a decentralized control in the MIMO framework to damp multiple swing modes through multiple HVDC devices. The controller is required to ensure that modal oscillations corresponding to each of the $n$ critical inter-area modes settle within $12.0 \mathrm{~s}$ for all the operating conditions considered.

For a MIMO system $G(s)$ with $m$-inputs and $m$-outputs, the overall control duty can be distributed amongst individual input-output pairs resulting in a diagonal $m$-input, $m$-output controller $K_{D}(s)$.

The problem can be mathematically formulated as follows:

$G_{i}(s) \triangleq\left[\begin{array}{c|c}A_{i} & B_{i} \\ \hline C_{i} & 0\end{array}\right] ; A \in \Re^{n \times n}, B \in \Re^{n \times m}, C \in \Re^{m \times n}$

The objective is to achieve a diagonal controller $K_{D}(s)$ such that it ensures stable system operation for $i$ operating conditions.

$$
\begin{aligned}
K_{D}(s) \triangleq & {\left[\begin{array}{c|c}
A_{k} & B_{k} \\
\hline C_{k} & D_{k}
\end{array}\right] } \\
& {\left[\begin{array}{cccc|cccc}
A_{k 1} & 0 & \cdot & 0 & B_{k 1} & 0 & \cdot & 0 \\
0 & A_{k 2} & \cdot & 0 & 0 & B_{k 2} & \cdot & 0 \\
\cdot & \cdot & \cdot & \cdot & \cdot & \cdot & \cdot & \cdot \\
0 & 0 & \cdot & A_{k m} & 0 & 0 & \cdot & B_{k m} \\
\hline C_{k 1} & 0 & \cdot & 0 & 0 & 0 & \cdot & 0 \\
0 & C_{k 2} & \cdot & 0 & 0 & 0 & \cdot & 0 \\
\cdot & \cdot & \cdot & \cdot & \cdot & \cdot & \cdot & \cdot \\
0 & 0 & \cdot & C_{k m} & 0 & 0 & \cdot & 0
\end{array}\right] }
\end{aligned}
$$

$A_{k j} \in \Re^{n_{k} \times n_{k}}, B_{k j} \in \Re^{n_{k} \times 1}, C_{k j} \in \Re^{1 \times n_{k}}, j=1,2, \cdots, m$

subject to an objective function $F$ such that:

$$
\begin{gathered}
\underset{K(s), \alpha_{i}}{F}=\sum_{p=1}^{n} \sum_{q=1}^{i}\left(\alpha_{\text {desired }}-\alpha_{p q}\right)^{2} \\
\sum_{i=1}^{m} \alpha_{i}=1
\end{gathered}
$$

This objective function ensures that the closed-loop damping ratios result in a maximum settling time of $12.0 \mathrm{~s}$ for $n$ critical inter-area modes taking into account $i$ probable operating conditions. The suffix $p$ and $q$ denote the mode number and the operating condition. $\alpha$ is the real part of the closed-loop eigen values. Given the non-linearity of the problem and lack of reasonable initial guess for the optimization variables, evolutionary optimization was considered here. Any evolutionary optimization technique could have been used to solve the problem. Here particle swarm technique was used.

\section{Decentralized Controller Design}

The closed-loop structure of the system is illustrated in Fig.11. The final controller is made up of 3 sub-controllers where each channel consists of only one gain $(K)$ and a maximum of four first-order lead-lag blocks terms, see Fig. 12, beside a standard low-pass noise filter $\left(T_{r}=0.01 \mathrm{~s}\right)$ and highpass washout blocks $\left(T_{w}=2.0 s\right)$. The structure and order of the compensators in each channel are chosen to reflect those of a standard PSS structure [1].

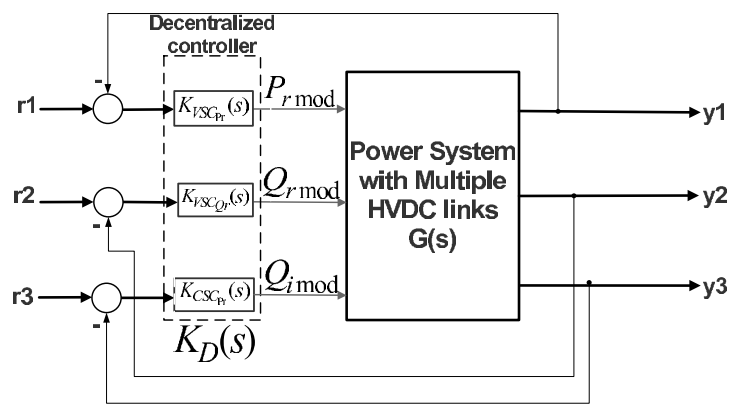

Fig. 11. Structure of the decentralized damping controller

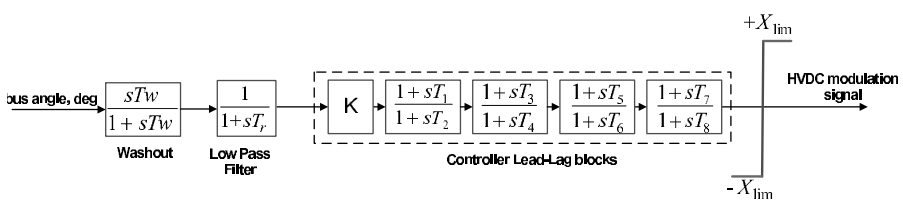

Fig. 12. Sub-controller structure for each input-output channel

TABLE II

DAMPING RATIOS AND FREQUENCIES OF CRITICAL MODES FOR LIGHT AND HEAVY LOADING CONDITIONS

\begin{tabular}{|c|c|c||c|c||c|c|}
\hline \multirow{2}{*}{$\begin{array}{c}\text { Loading } \\
\text { Condition }\end{array}$} & \multicolumn{2}{|c||}{ AC system } & \multicolumn{2}{c||}{ with Multiple HVDC } & \multicolumn{2}{c|}{ with POD } \\
\cline { 2 - 7 } & $\zeta, \%$ & $f, \mathrm{~Hz}$ & $\zeta, \%$ & $f, \mathrm{~Hz}$ & $\zeta, \%$ & $f, \mathrm{~Hz}$ \\
\hline \hline \multirow{3}{*}{ Light } & 8.8 & 0.42 & 8.0 & 0.36 & 16.0 & 0.36 \\
\cline { 2 - 7 } & 5.6 & 0.51 & 9.0 & 0.48 & 16.0 & 0.38 \\
\cline { 2 - 7 } & 3.7 & 0.63 & 3.0 & 0.63 & 9.0 & 0.65 \\
\hline \hline \multirow{3}{*}{ Heavy } & 12.4 & 0.28 & 10.0 & 0.27 & 17.0 & 0.31 \\
\cline { 2 - 7 } & 8.8 & 0.34 & 13.5 & 0.35 & 17.0 & 0.32 \\
\cline { 2 - 7 } & 5.9 & 0.52 & 6.1 & 0.52 & 10.0 & 0.53 \\
\hline
\end{tabular}

\section{RESULTS AND DISCUSSION}

\section{A. Linear Analysis}

The location of the open loop poles (excluding the higher frequency modes) is shown in Figs 7 and 8. With damping control, the three critical poles are shifted left towards the reference desired settling time of $12.0 \mathrm{sec}$. The closed-loop settling times, shown in Fig. 13, are within the desired 12.0 $\mathrm{s}$ target, indicated by the horizontal line which demonstrates that the design objective has been satisfied. The dynamic 


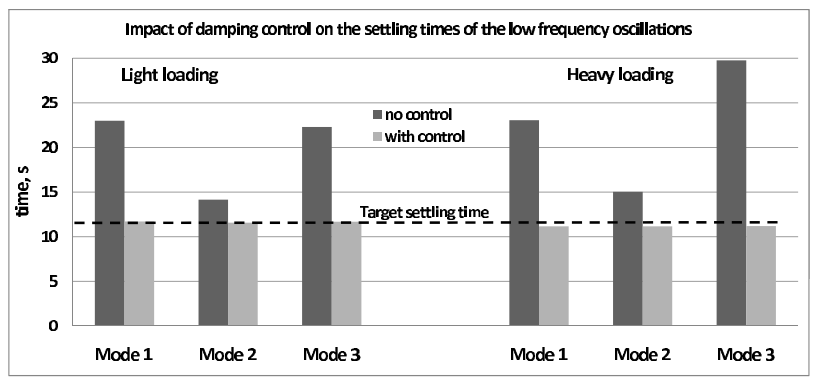

Fig. 13. Open and closed loop settling time ( in seconds) for the three (mode 1 , mode 2 , mode 3 ) modal oscillations under two extreme loading conditions

performance in the presence of non-linearities is demonstrated through time domain simulations in the next section.

In this section, time domain simulation results obtained directly from DIgSILENT PowerFactory are presented for the two extreme operating conditions. The dynamic performance of the system with and without control is studied here. A threephase self-cleared solid fault is simulated for 5 cycles $(100 \mathrm{~ms})$ between area 1 and area 2 where the flows are most severe. Time domain results are presented in Figs 14 and 15.

\section{B. Non-linear Simulation in DIgSILENT PowerFactory}

In all cases the desired settling time of $12.0 \mathrm{~s}$ is achieved by supplementary control loops modulating the active power order of CSC and the active \& reactive power for the VSC converter stations. The open loop results are shown in blue and the closed loop response are shown in red. Figs 14 and 15 $(a),(b)$ show the angular separation between generators G502 and G503 respectively, with reference to the slack generator G101. subplot $(c)$ exhibits power flow in line 315-509. (d) shows the outputs of the POD controllers required to damp the power oscillations as seen in $(a, b, c)$.

1) Light loading condition: Interestingly, the control effort required with modulation of $Q_{r}$ is much higher $P_{r}$ modulation. Subplots $(e, f)$ demonstrate the ability of the CSC-HVDC to regain its steady-state condition after the fault has cleared. The VSC current controls $I_{d i} \& I_{q r}$ are seen to change in subplots $(g, h)$ resulting from variations in the DC link dynamics.

2) Heavy Loading Condition : To test the adequacy of the decentralized controller for a different loading condition, the dynamic performance of the system was evaluated for the heavy loading condition where in open-loop the swing amplitude are appreciably larger. Under such conditions, the control efforts demanded from the damping controller is expected to be more significant than those of the light loading condition, if the design requirement of $12.0 \mathrm{~s}$ settling times is to be satisfied. This is evident in Fig. $15(d)$ where $Q_{r}$ is seen to momentarily violate its respective limits.

\section{CONCLUSION}

Efectiveness of multiple HVDC links - both CSC and VSC - in improving the dynamic performance of an AC system has been demonstrated through a case study on the Australian equivalent system with three critical inter-area modes. Subspace-based multi-input-multi-output (MIMO) system identification was used to estimate and validate linearized

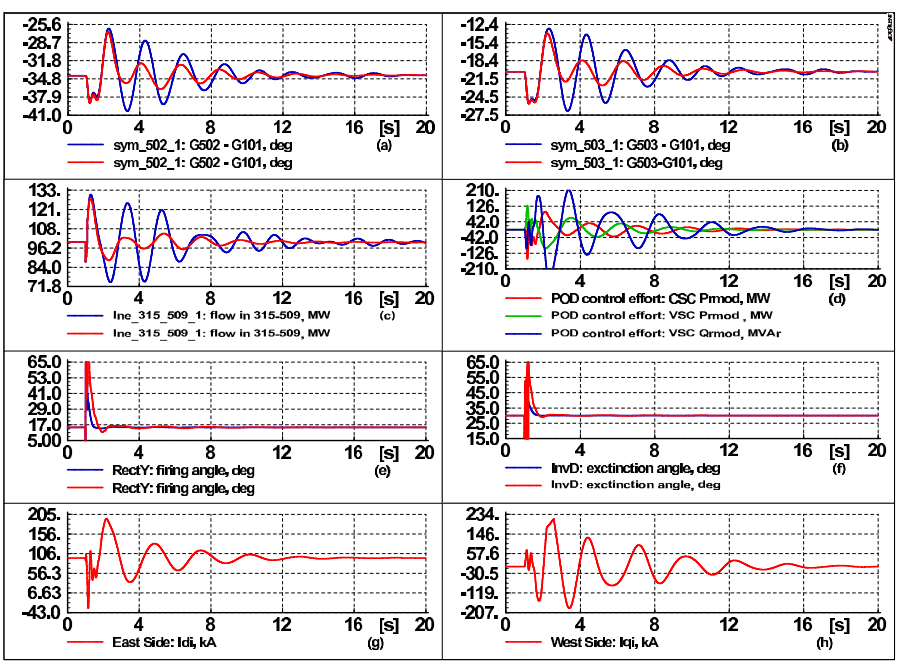

Fig. 14. Dynamic performance of the system under light loading condition following a 3-phase self cleared fault at line 102-217 for $100 \mathrm{~ms}$. Blue represents without control and red plots show with control.

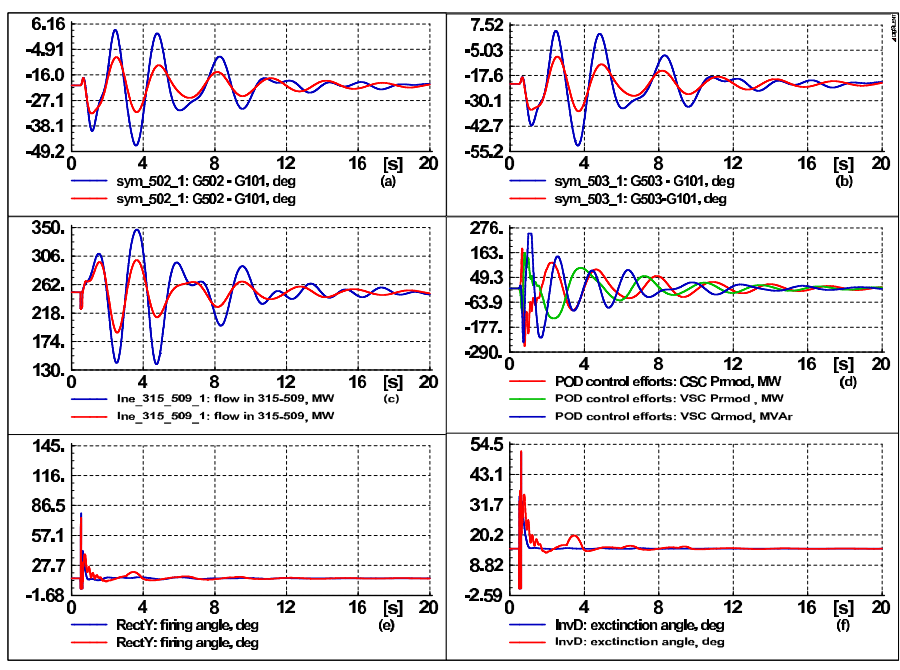

Fig. 15. Dynamic performance of the system under heavy loading condition following a 3-phase self cleared fault at line 102-217 for $100 \mathrm{~ms}$. Blue represents without control and red plots show with control.

state-space models through pseudo random binary sequence (PRBS) probing in DIgSILENT PowerFactory. Choice of appropriate input-output combinations was done through modal residue analysis taking note of not only the magnitudes but also the phase angles. The decentralized control design was formulated as an optimization problem and solved using an evolutionary technique. The results were validated through linear analysis and non linear simulation in DIgSILENT for two extreme (light and heavy loading) operating conditions.

\section{REFERENCES}

[1] P. Kundur, Power system stability and control, ser. The EPRI power system engineering series. New York; London: McGraw-Hill, 1994.

[2] J. Arrillaga, Y. H. Liu, and N. R. Watson, Flexible power transmission: the HVDC options. John Wiley, 2007.

[3] R. Ramos, L. Alberto, and N. Bretas, "A new methodology for the coordinated design of robust decentralized power system damping controllers," IEEE Transactions on Power Systems, vol. 19, no. 1, pp. 444-454, 2004. 
[4] A. R. Messina, O. Begovich, J. H. Lpez, and E. N. Reyes, "Design of multiple facts controllers for damping inter-area oscillations: a decentralised control approach," International Journal of Electrical Power \& Energy Systems, vol. 26, no. 1, pp. 19 - 29, 2004.

[5] C. Schauder and H. Mehta, "Vector analysis and control of advanced static var compensators," IEE Proceedings on Generation, Transmission and Distribution, vol. 140, no. 4, pp. 299-306, 1993.

[6] D. Vowels and M. Gibbard. (2008) PSDG contribution to the IEEE benchmark systems for stability controls task force. Internet draft. [Online]. Available: http://www.eleceng.adelaide.edu.au/groups/PCON/PowerSystems/IEEE/Benchmark

[7] V. K. Sood, HVDC and FACTS controllers: applications of static converters in power systems. Boston; London: Kluwer Academic Publishers, 2004.

[8] T. C. Hsia, System identification: least-squares method. [S.1.]: Lexington Books, 1977.

[9] J. F. Hauer, W. A. Mittelstadt, K. E. Martin, J. W. Burns, H. Lee, J. W. Pierre, and D. J. Trudnowski, "Use of the wecc wams in wide-area probing tests for validation of system performance and modeling," IEEE Transactions on Power Systems, vol. 24, no. 1, pp. 250-257, 2009.

[10] P. v. Overschee and B. L. R. d. Moor, Subspace identification for linear systems : theory, implementation, applications. Boston ; London: Kluwer Academic, 1996.

[11] S. Ray, B. Chaudhuri, and R. Majumder, "Appropriate signal selection for damping multi-modal oscillations using low order controllers," in proceedings of IEEE Power Engineering Society General Meeting, 2008, Pittsburgh, 2008. 